\title{
Spatiotemporal variations in ischemic heart disease mortality and related risk factors in China between 2010 and 2015: a multilevel analysis
}

Baohua Wang ${ }^{1}$, Peiyao Li $i^{2}$, Fengdie He${ }^{1}$, Yuting Sha ${ }^{1}$, Xia Wan ${ }^{3^{*}}$ and Lijun Wang ${ }^{1 *}$

\begin{abstract}
Background: To explore the relationship between geographical differences of mortality and related risk factors in ischemic heart disease (IHD) in China.

Methods: Data were collected from the nationally representative China Mortality Surveillance System to calculate annual IHD mortality counts (2010-2015). Descriptive analysis was used to analyze the IHD mortality among Chinese population from 2010 to 2015. Negative binomial regression was used to investigate potential spatiotemporal variation and correlations with age, gender, urbanization, and region.

Results: The overall IHD mortality was 221.17/100,000, accounting for 1.51 million deaths in 2015. The standardized IHD mortality rate increased by 5.51\% from 2010 to 2015 among people aged 40 years and older. Multilevel analysis indicated significant differences in gender, regions, and age. High urbanization rate (risk ratio $[R R]=0.728,95 \%$ confidence interval $[\mathrm{Cl}]=(0.631,0.840))$ and average high-density lipoprotein $(\mathrm{HDL})(\mathrm{RR}=0.741,95 \% \mathrm{Cl}: 0.616,0.891)$ were negatively associated with IHD mortality. IHD mortality was significantly higher in populations with a low rate of medical insurance coverage ( $R R=1.218,95 \% \mathrm{Cl}: 1.007,1.473)$, as well as the average body mass index (BMI) $(\mathrm{RR}=$ $1.436,95 \% \mathrm{Cl}: 1.135,1.817)$ and systolic blood pressure (SBP) $(\mathrm{RR}=1.310,95 \% \mathrm{Cl}: 1.019,1.684)$. While the relationship with current smoking rate, excessive intake of red meat, insufficient vegetable or fruits intake didn't show the statistical significance. The negative correlation between the average sedentary time and IHD mortality was not conclusive due to the possible deviation of the data.

Conclusions: The mortality of IHD showed an upward trend for people aged 40 years and older in China during 2010-2015, which should be paid attention to. Therefore, some risk factors should be controlled, such as SBP, overweight/obesity. HDL is a protective factor, as well as higher urbanization rate, family income level, and medical insurance coverage.
\end{abstract}

Keywords: Ischemic heart disease, Risk factors, Multilevel statistical model

\footnotetext{
*Correspondence: xiawan@ibms.pumc.edu.cn; 2496393510@qq.com

${ }^{3}$ Institute of Basic Medical Sciences Chinese Academy of Medical Sciences,

School of Basic Medicine Peking Union Medical College, Beijing, China

${ }^{1}$ National Center for Chronic and Noncommunicable Disease Control and

Prevention, Chinese Center for Disease Control and Prevention, 27 Nanwei

Road, Xicheng District, Beijing 100050, P.R. China

Full list of author information is available at the end of the article
}

C C The Author(s). 2021 Open Access This article is licensed under a Creative Commons Attribution 4.0 International License, which permits use, sharing, adaptation, distribution and reproduction in any medium or format, as long as you give appropriate credit to the original author(s) and the source, provide a link to the Creative Commons licence, and indicate if changes were made. The images or other third party material in this article are included in the article's Creative Commons licence, unless indicated otherwise in a credit line to the material. If material is not included in the article's Creative Commons licence and your intended use is not permitted by statutory regulation or exceeds the permitted use, you will need to obtain permission directly from the copyright holder. To view a copy of this licence, visit http://creativecommons.org/licenses/by/4.0/ The Creative Commons Public Domain Dedication waiver (http://creativecommons.org/publicdomain/zero/1.0/) applies to the data made available in this article, unless otherwise stated in a credit line to the data. 


\section{Background}

Ischemic heart disease (IHD) has caused $30 \%$ of total annual deaths, which become the leading cause of death and a major economic burden worldwide [1,2]. Two out of five deaths were due to cardiovascular disease, especially in rural areas [3]. IHD has imposed a heavy burden on families and society overall, and has become a major public health issue.

The multilevel model (MLM), also called linear mixed model or hierarchical linear model, has become increasingly popular in public health for analyzing data with repeated measurements or data organized in nested levels [4]. The advantages of it are as follow: (i) It could effectively estimate the regression coefficient, (ii) provide the confidence interval $(\mathrm{CI})$ and the hypothesis test by using the aggregation information and the correct standard error; (iii) and introduce the co-variable at any level to explore the effects of all variables at any level for researchers [5].

The distribution of IHD in China has spatial and temporal differences. For example, from 1991 to 2000, counties with high IHD mortality were located in northwest, north, and northeast China, while from 2004 to 2009, the high mortality rate areas expanded to the north, central and southern regions. Counties with an increase in IHD mortality of more than $100 \%$ are concentrated in the Northeast and South [6]. In 2015, the highest agestandardized mortality rate per 100,000 people of IHD was Heilongjiang Province (187.4, 95\% CI: 161.6-217.5), and the lowest was Shanghai (44.2, 95\% CI: 37.0-53.1). Geographically, the age-standardized death and DALY rate in the southern provinces were lower than those in the northern provinces, especially in the southeastern coastal provinces [7]

Therefore, the data from Disease Surveillance Systems (DSP), with nationally representative, was used to investigate temporal trends and geographical variations in IHD mortality in China from 2010 to 2015. The key objectives of this study are as follows: (i) to investigate the levels and characteristics of IHD mortality across China from 2010 to 2015, (ii) to explore whether there are geographical differences in IHD mortality rates and to determine whether those differences vary over time, and (iii) to establish a multilevel model for the analysis of factors affecting IHD in the Chinese population.

\section{Methods}

\section{Data source}

The International Classification of Diseases 10 (ICD-10) was used to define IHD mortality in the China Mortality Surveillance System. The relevant codes included I20I25(Angina pectoris, Acute myocardial infarction, Subsequent myocardial infarction, Chronic ischemic heart disease). More information on DSP system were described elsewhere [8]. In general, it comprises 161 counties or districts across all 31 provinces, municipalities, and autonomous regions in Mainland China, providing coverage of approximately 73 million people [9]. County- and district-level (i.e., DSP) population counts cross- classified by 5-year age group and gender were obtained from the Chinese census in 2000 and 2010. These counts were used as a reference population for calculating the age-standardized mortality rates of IHD and in statistical models. The total population for each DSP in the years 2010-2015 was then estimated assuming an exponential growth across the time period, in line with the methodology used in the estimation of mortality rates in China in the Global Burden of Disease Study (GDB) [10].

In total, 161 DSPs were classified as 'rural' $(n=97)$ or 'urban' ( $n=64)$ according to administrative characteristics. Meanwhile, according to their regional affiliation by the China National Bureau of Statistics [11], all the DSPs were classified into seven regions [12].

In addition, person-level data from the China Chronic Disease Risk Factor Surveillance (CDRFS) in 2013 was used to estimate the prevalence of risk factors at the DSP level. Detailed design and methods were described elsewhere [13]. CDRFS was a cross-sectional populationbased survey with national representative, which included 98,658 adult participants from all 161 DSP sites by using a complex, multistage, probability sampling design. A questionnaire including information on demographic characteristics, medical history, and lifestyle factors was conducted by trained interviewers.

\section{Model information}

Based on the characteristics of death data, negative binomial regression was selected to use for over-dispersion of the mortality counts [14]. An initial model included fixed effects for age group, gender, and year. A threelevel model was fitted, with the DSP/gender/5-year age group cross-classification at Level 1, county at Level 2, and province at Level 3. The natural logarithm of the equivalently classified denominator counts was fitted as an offset to account for local population distribution. The behavioral risk factor variables and socioeconomic indicators are added to the model one by one to calculate the percentage variance change (PCV) of the variance between the province and county levels. The relative risk (RR) and 95\% confidence interval $(\mathrm{CI})$ of all fixed effect parameters can be obtained through the model.

In this study, each behavioral risk factor index and socio-economic index were divided into three grades: low, medium, and high according to tertiles. Specific indicators included: urbanization rate, current smoking rate, excessive intake of red meat, insufficient intake of 
fruits and vegetables, average sedentary time, average body mass index (BMI), average systolic blood pressure (SBP), fasting blood glucose (FBG) average level, highdensity lipoprotein (HDL) average level, average total cholesterol level, triglyceride level, non-medical insurance ratio, family income level.

\section{Statistical analysis}

The median rate ratios (MRRs) was calculated by fitting the variance of random effects. MRRs is calculated using the same equation as the median odds ratio (MOR) and has a similar interpretation; that MRRs equal to 1 suggests no geographic variation in the outcome variable, whereas values above 1 indicate the necessity of taking context into account [15]. A common application of multilevel models is to apportion the variance in the response according to the different levels of the data [16]. Much interest is focused on the amount of variation attributable to the high level, because this provides a quantitative estimation of "where the action lies". Such a measure in simple variance components, continuous response, variance partition coefficient (VPC) is often referred to as the "intra-class correlation, ICC". In the two-level variance component model, VPC represents the proportion of level 2 (high level) variance to the total variance:

$$
V P C=\frac{\sigma_{u 0}^{2}}{\sigma_{e}^{2}+\sigma_{u 0}^{2}}
$$

where $\sigma_{u 0}^{2}$ is the variance related to the level 2 (high level), $\sigma_{e}^{2}$ is the level1(low level). More statistical notations are referred elsewhere $[17,18]$.

All fixed effect parameters were exponentiated to RRs and 95\% CIs. All statistical analyses were conducted in MLwIN v2.30.

\section{Results}

\section{IHD mortality in the Chinese population from 2010 to} 2015

The IHD crude mortality rates was 221.17 per 100,000 in 2015 and increased by $23 \%$ (from 88.16 to 108.74 per 100,000) from 2010 to 2015 in the overall population (Table 1). The age-standardized mortality rates of IHD among people aged 40 years and older increased $5.51 \%$ (from 145.95 to 153.99 per 100,000) from 2010 to 2015(Table 2). Gender differences were also observed, with IHD mortality increasing more slowly for males $(5.18$ per 100,000$)$ than females $(9.51$ per 100,000$)$. The disparity between urban and rural residence was notable. IHD mortality rates during the study period decreased by $1.53 \%$ (from 133.89 to 132.36 ) in urban areas but increased by $14.88 \%$ (from 154.79 to 169.67 ) in rural areas.

The mortality rate was apparently different between males and females, and between urban and rural regions during the study period (Fig. 1). Age-standardized IHD mortality rates in females and urban areas were lower than in males and rural areas. In this study, the superposition effect of gender and urban/rural areas in agestandardized mortality rates showed that the IHD mortality rates were highest in rural males and were lowest in urban females. In urban areas, the agestandardized IHD mortality rates decreased $2.00 \%$ for males (from 152.97/ 100,000 in 2010 to 149.91/ 100,000 in 2015), and $0.6 \%$ for females (from $115.12 / 100,000$ in 2010 to $114.43 / 100,000$ in 2015), respectively. However, in rural areas, the age-standardized IHD mortality rates increased 6.17\% for males (from 183.66/ 100,000 in 2010 to $195.00 / 100,000$ in 2015) and $13.8 \%$ for females (from $128.16 / 100,000$ in 2010 to $144.92 / 100,000$ in 2015), respectively (see Fig. 1a, b).

As shown in Table 2, mortality rates were highest in the central region and lowest in the west. Mortality rates in the eastern region primitively increased and then decreased in total (from 140.93/100,000 to 140.83/100, 000). Nevertheless, mortality rates in the western and central region increased over time (from 116.05/100,000 to $135.71 / 100,000$, from $173.01 / 100,000$ to $184.09 / 100$, 000, respectively). The increasing slope was highest from 2010 to 2013 (Fig. 1c).

Gender and region stratified analyses showed the mortality rates were different. For males, the mortality rates in the eastern region showed a downward trend (from $165.17 / 100,000$ to $164.21 / 100,000)$. However, the agestandardized IHD mortality rates increased by $6.40 \%$ from $173.01 / 100,000$ to $184.09 / 100,000$ in the central region, and it increased by $16.94 \%$ from $116.05 / 100,000$ to $135.71 / 100,000$ in the western region. For females, in

Table 1 Crude mortality of IHD in people over 40 years old in different regions from 2010 to $2015(1 / 100,000)$

\begin{tabular}{|c|c|c|c|c|c|c|c|c|}
\hline & Urban & Rural & Male & Female & East & Central & West & Total \\
\hline 2010 & 183.04 & 208.87 & 212.24 & 183.60 & 201.69 & 227.20 & 152.40 & 197.99 \\
\hline 2011 & 185.65 & 212.93 & 216.76 & 187.06 & 199.25 & 234.41 & 157.91 & 201.71 \\
\hline 2012 & 190.13 & 235.96 & 230.59 & 202.48 & 206.65 & 250.34 & 184.24 & 216.59 \\
\hline 2013 & 202.31 & 248.77 & 244.87 & 213.87 & 220.27 & 263.63 & 195.52 & 229.39 \\
\hline 2014 & 194.78 & 233.20 & 229.40 & 203.91 & 209.10 & 245.55 & 189.70 & 216.67 \\
\hline 2015 & 198.73 & 236.58 & 233.56 & 208.80 & 215.34 & 254.34 & 186.47 & 221.17 \\
\hline
\end{tabular}


Table 2 Standardized mortality of IHD in people over 40 years of age in different regions from 2010 to 2015 (1/100,000)

\begin{tabular}{|c|c|c|c|c|c|c|}
\hline Year & 2010 & 2011 & 2012 & 2013 & 2014 & 2015 \\
\hline \multicolumn{7}{|l|}{ Urban } \\
\hline Male & 152.97 & 153.31 & 154.50 & 159.38 & 150.23 & 149.91 \\
\hline Female & 115.12 & 113.28 & 115.86 & 120.07 & 116.44 & 114.43 \\
\hline Total & 133.89 & 133.11 & 135.17 & 139.83 & 133.50 & 132.36 \\
\hline \multicolumn{7}{|l|}{ Rural } \\
\hline Male & 183.66 & 184.82 & 202.73 & 212.04 & 195.63 & 195.00 \\
\hline Female & 128.16 & 131.13 & 145.43 & 152.17 & 142.18 & 144.92 \\
\hline Total & 154.79 & 156.88 & 173.15 & 181.49 & 168.30 & 169.67 \\
\hline \multicolumn{7}{|l|}{ Urban+rural } \\
\hline Male & 170.59 & 171.62 & 181.87 & 189.37 & 175.38 & 175.77 \\
\hline Female & 122.56 & 123.69 & 132.81 & 138.48 & 130.81 & 132.07 \\
\hline Total & 145.95 & 147.04 & 156.93 & 163.76 & 152.99 & 153.99 \\
\hline \multicolumn{7}{|l|}{ East } \\
\hline Male & 165.17 & 160.66 & 166.15 & 171.62 & 161.97 & 164.21 \\
\hline Female & 118.50 & 116.91 & 120.17 & 125.53 & 117.53 & 117.93 \\
\hline Total & 140.93 & 138.08 & 142.53 & 148.34 & 139.42 & 140.83 \\
\hline \multicolumn{7}{|l|}{ Central } \\
\hline Male & 202.93 & 206.87 & 216.34 & 224.24 & 204.83 & 208.63 \\
\hline Female & 144.46 & 148.01 & 158.15 & 165.01 & 155.29 & 159.37 \\
\hline Total & 173.01 & 176.67 & 186.84 & 194.36 & 179.93 & 184.09 \\
\hline \multicolumn{7}{|l|}{ West } \\
\hline Male & 135.27 & 139.28 & 158.79 & 169.69 & 158.3 & 151.91 \\
\hline Female & 97.41 & 99.11 & 117.05 & 121.42 & 120.61 & 118.93 \\
\hline Total & 116.05 & 118.84 & 137.76 & 145.60 & 139.62 & 135.71 \\
\hline
\end{tabular}

total, the age-standardized IHD mortality rates slightly decreased from $118.50 / 100,000$ to $117.93 / 100,000$ in the eastern region. The mortality of western and central regions increased in total (from 97.41/100,000 to 118.93/ 100,000 , from $144.46 / 100,000$ to $159.37 / 100,000$, respectively), while the mortality rates were lower after 2013 (Fig. 1).

\section{IHD mortality in the Chinese population from 2010 to 2015 in multilevel models}

Multilevel model 1 (Table 3) indicates that age and the gender-adjusted IHD mortality increased between 2010 and 2015 ( $R R=1.016,95 \%$ CI: $1.012,1.020)$, and it increased with age $(\mathrm{RR}=1.839,95 \% \mathrm{CI}: 1.835,1.842)$. IHD mortality was lower among females $(\mathrm{RR}=0.622,95 \% \mathrm{CI}$ : $0.612,0.632)$. The provincial and DSP intercept variance was statistically significant $(\mathrm{MRRp}=1.514$ and $\mathrm{MRRd}=$ 1.459), which indicated the necessity of using multilevel models. IHD mortality rate was explained by adjustment for region in model 2. Compared to the northern region, IHD mortality rate was significantly lower in the east $(R R=0.435,95 \%$ CI: $0.307,0.616)$, southwest $(R R=$
0.360, 95\% CI: $0.246,0.525)$, and south $(R R=0.512,95 \%$ CI: $0.329,0.795)$. The provincial and DSP intercept variance was significantly different $(\mathrm{MRRp}=1.258$ and MRRd $=1.430$ ). To explore the role of socioeconomic factors on IHD mortality, the urbanization rate of each DSP was added in multilevel model 3. Comparing with low urbanization rate areas, the RR and 95\% CI of the areas with a high urbanization rate was $0.728(0.631$, $0.840)$.

Lifestyle factors (smoking, red meat intake, vegetable and fruit deficiency, sedentary time) were included in the multilevel model 4 . The results showed that smoking, red meat intake, vegetable and fruit deficiency, and sedentary time did not have statistically significant. Compared to model 3, model 4 increased approximately $0.7 \%$ of the DSP-level PCV in IHD mortality. In model 5 , which incorporated the six metabolic risk factors (average BMI, SBP, mean FBG, HDL cholesterol, cholesterol level and triglyceride level), only average BMI and SBP were positively associated with IHD mortality ( $R R=$ 1.495, 95\% CI: $1.172,1.906, \mathrm{RR}=1.294,95 \%$ CI: 1.007 , 1.663). Moderate and high HDL cholesterol levels were negatively correlated with IHD mortality $(\mathrm{RR}=0.830$, 95\%CI:0.706, 0.977, RR $=0.746, \quad 95 \% \mathrm{CI}: \quad 0.619,0.899$, respectively). In order to know the role of personal economic situation on the IHD mortality, health insurance rate and family income level were added into multilevel model 6. A low health insurance rate was positively associated with the IHD mortality ( $R R=1.218,95 \% \mathrm{CI}$ : $1.007,1.473)$, indicating the importance of health insurance coverage expansion. The relationship between family income level and IHD mortality is not obvious $(\mathrm{RR}=0.886,95 \% \mathrm{CI}: 0.741,1.059)$. Moderate sedentary time was negatively correlated with IHD mortality $(\mathrm{RR}=$ $0.851,95 \%$ CI: $0.739,0.980$ ) in model 6 , but it showed no correlation with IHD mortality in model 4 and 5 . In model 6, approximately 94.7 and $38.1 \%$ of the provincial-level and the DSP-level variation, respectively, in IHD mortality was explained by considering behavioral risk factors and socioeconomic factors.

The VPC was reduced when lifestyle variances were introduced in the model, which suggested that some part of the contextual phenomenon related to IHD on monitoring point was attributable to provincial deprivation.

\section{Discussion}

Cardiovascular and cerebrovascular diseases are the leading cause of death worldwide. The results of this study showed that the IHD mortality rate was 108.74/ 100,000 in 2015, accounting for $39.74 \%$ of cardiocerebrovascular disease. IHD mortality in the males was higher than that in the females. For people aged 40 years and older, the crude IHD mortality rate was $221.17 / 100$, 000 in 2015. Furthermore, the results of our analysis 

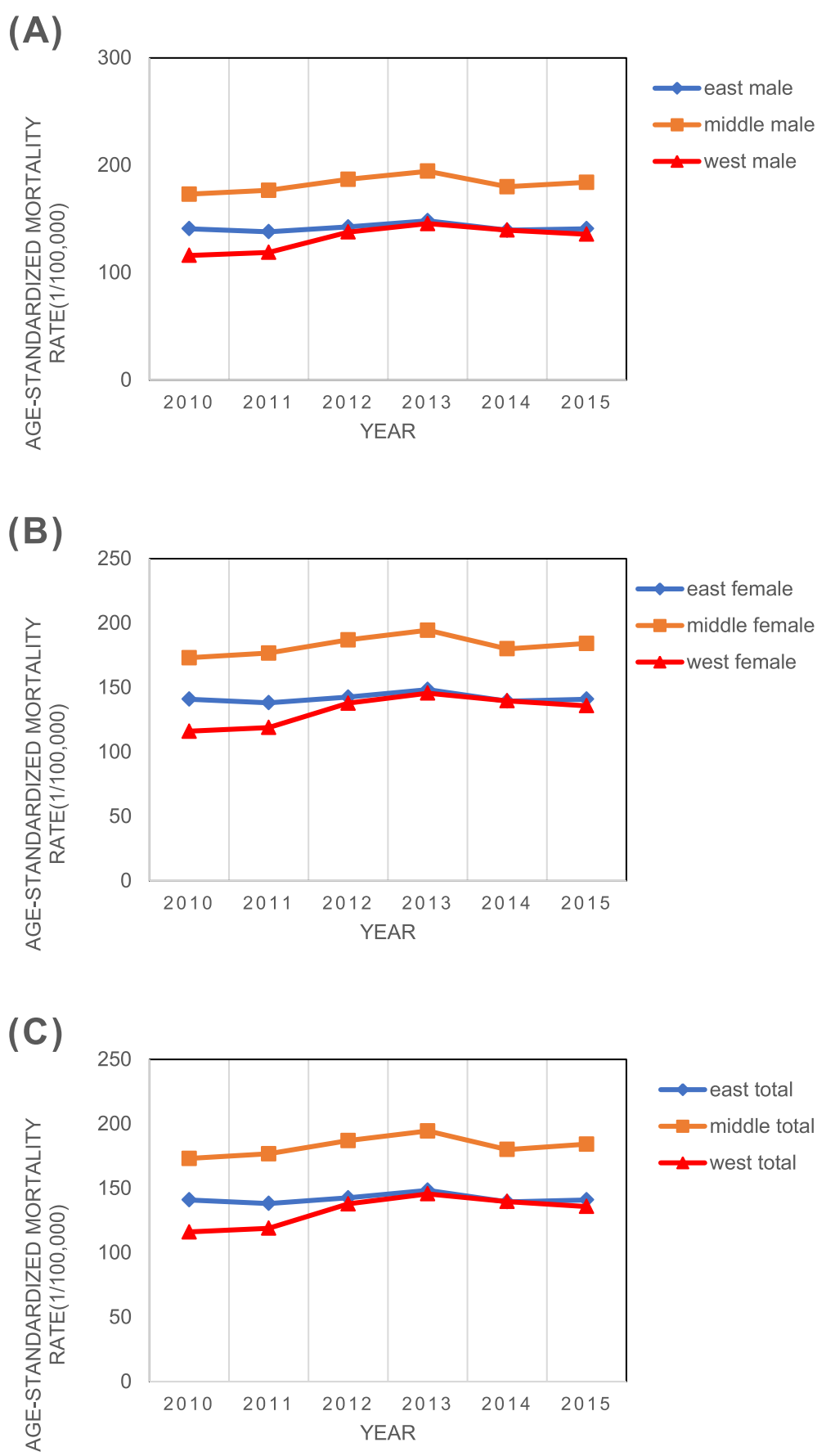

Fig. 1 Age-standardized mortality rates of IHD in people over 40 years of age in different regions from 2010 to $2015(1 / 100,000)$

showed that the crude IHD mortality rate was higher in the rural males and in the central region, compared to urban females in other regions. This might be a result of stress and lifestyle. For example, males tend to have bad habits such as drinking [19] and smoking [20]. Regional differences might be related to several factors, such as the level of socioeconomic status, availability and affordability of health services, consciousness of prevention, urbanization, economic growth, and epidemiological transition on cardiovascular health [21, 22].

Our results show that the crude IHD mortality rate increased by $23.34 \%$ during the $2010-2015$. After eliminating the influence of the aging population, the standardized IHD mortality rate showed an upward 


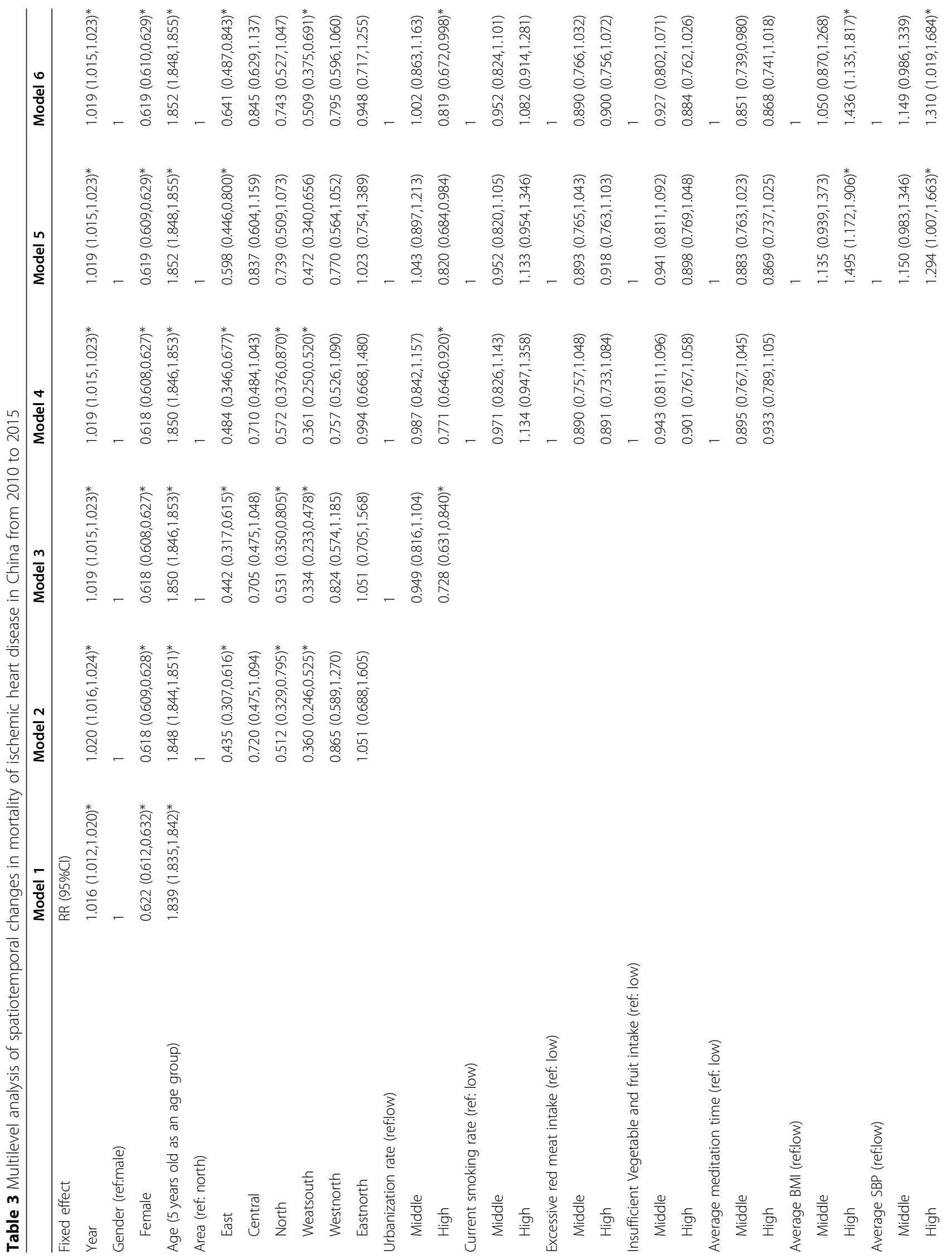




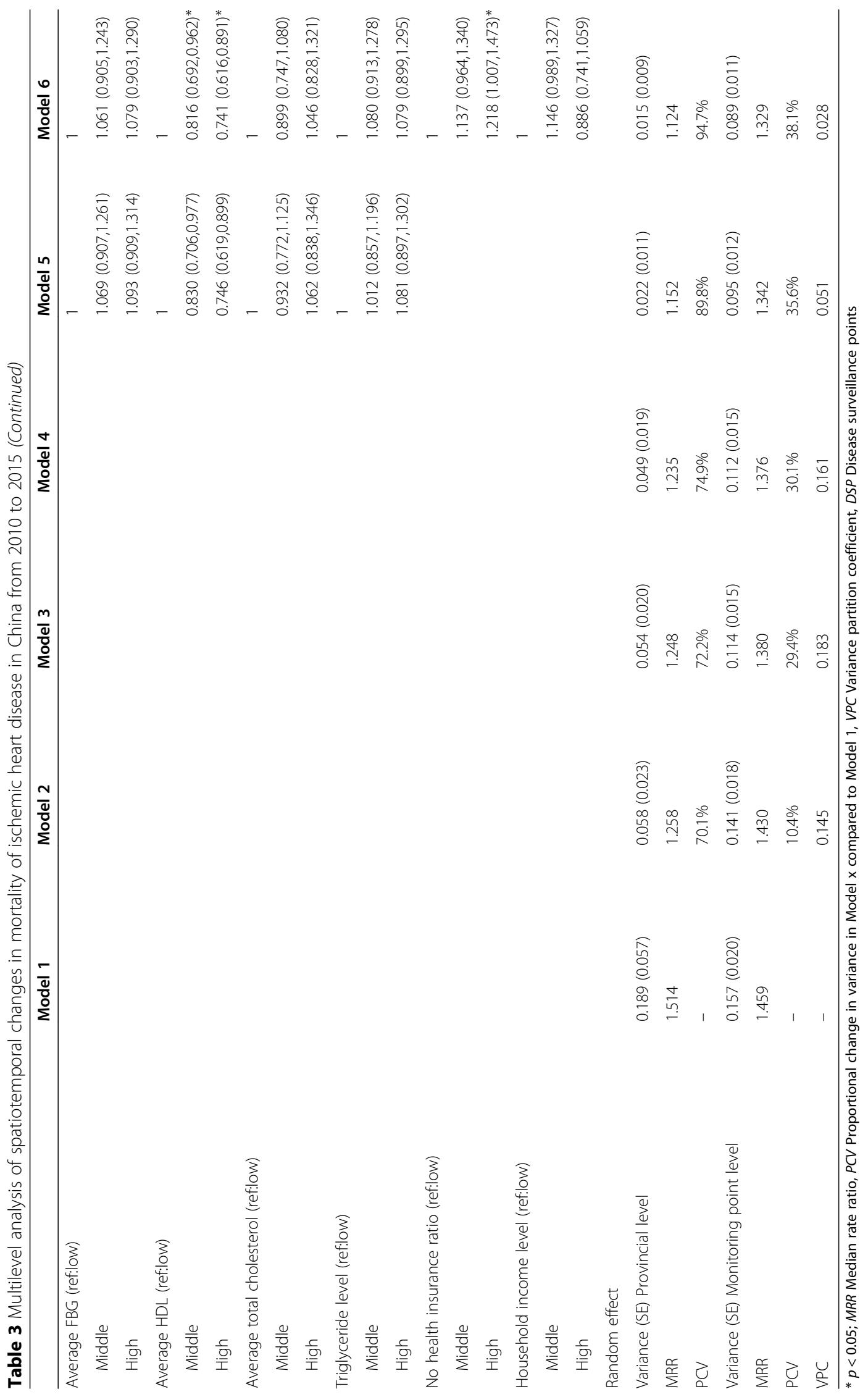


trend. The age-standardized IHD mortality among people aged 40 years and older also increased from 2010 to 2015 [10].

Many large prospective cohort studies have shown that hypertension, hyperlipidemia, diabetes, smoking, and obesity are the main risk factors for IHD [23]. Elevated blood pressure is the most important determinant risk factors for stroke [24]. Unhealthy lifestyles such as smoking, drinking, high salt diet, dietary imbalance, and lack of physical activity can directly or indirectly affect the incidence and mortality rate of IHD [25]. In this study, a multilevel model was used to study the relationship between the regional and the other related risk factors on the IHD mortality rate.

Study have found that mortality from IHD is associated with socioeconomic status (low education, poverty, working and living conditions). Developed regions, with greater healthcare financing and efficient primary health care, have lower mortality rates [26]. The results of our multilevel model showed that a high urbanization rate was negatively correlated with the IHD mortality rate; and the higher the urbanization rate, the lower IHD the mortality rate. The rapid development of China's economy, great changes have taken place in people's diet, especially in the urban areas. They eat more meat and milk, high calorie foods, foods high in fat and protein, and less whole grains and vegetables. These lifestyle changes increase the risk of IHD, which is consistent with results from individual-level prospective studies [27]. However, moderate intake of fruits, vegetables, and beans can reduce the risk of death from cardiovascular disease [28]. The level of medical treatment in urban regions, the health infrastructure, institutional care, palliative care, and hospice care have been significantly improved. The results of this article also support that the conclusion that the IHD mortality rate is basically stable in the urban and eastern regions, while rising in rural and western areas.

Many studies have confirmed that the risk factors of metabolism are closely related to the pathogenesis of cardio-cerebrovascular diseases. The prominent risk factors are hypertension, hyperlipidemia, diabetes, overweight/obesity [29]. Our study showed that high BMI level and high SBP level were positively correlated with IHD, with RRs of 1.495 and 1.294, respectively. Moderate HDL level and high HDL level were negatively correlated with IHD, with RRs of 0.830 and 0.746, respectively.

We found that BMI was positively correlated with IHD mortality, which is consistent with the prospective study [30]. A cohort study in South Korea showed that $\mathrm{SBP}>120 \mathrm{mmHg}$ and DBP $>70 \mathrm{mmHg}$ had a trend of having increased CVD incidences [31]. However, there is no statistical significance for the relationship between the prevalence of hypertension and IHD mortality. This might be related to the diagnostic basis of hypertension. Many studies have shown that pulse pressure is an independent factor for cardio-cerebrovascular diseases. HDL is also associated with IHD mortality. A comparison of the average HDL-C levels between Korean and American populations was conducted in order to estimate the optimal HDL-C threshold for predicting cerebrovascular accidents and IHD [32].

In recent years, as the prevalence of IHD increased, the medical expenses of IHD has grown. Because of the high cost of treating IHD in hospital, an increase in average family income will be beneficial to the reduction of IHD mortality. The results of our study support the conclusion that the IHD mortality rate was significantly higher in those without adequate medical insurance (RR $1.218,95 \%$ CI: $1.007,1.473)$. Thus, expanding the proportion of those with health insurance benefits will decrease IHD mortality.

In addition to metabolic factors, smoking, drinking, inadequate physical activity, unhealthy diet and other behavioral factors have been proved to have an impact on the incidence of IHD and its mortality rate in many studies. We found that smoking, red meat intake, and insufficient ingestion of vegetables and fruits did not have a significant effect on IHD mortality rate. In the analysis of the reasons, on the one hand, when we adopt the multilevel analysis, the behavioral factors are in the level of the monitoring point, which does not reflect the specific situation of the case. Furthermore, quality control measures were not consistent, leading to uneven data and recall bias. To ensure the accuracy of our data, we provided routine training to coding staff and had a regular checking and verification process to avoid coding errors.

In addition, our study found that the average sedentary time had a negative correlation with IHD mortality among people aged 40 years and over. A cohort study abroad also found that difference in risk of IHD was not observed between sedentary and non-sedentary employees [33]. However, a Canadian study [34] found that sedentary lifestyles were associated with cardiovascular disease and the incidence and mortality of cancers, especially type- 2 diabetes. Zhou et al. [35] studied the relationship between sedentary time and cardiocerebrovascular disease in Beijing, and they found that sedentary lifestyle was significantly associated with fasting blood glucose, triglycerides, cholesterol, and LDL.

An important strength of this study is that the data on IHD in this article comes from the long-term monitoring data of 161 DSPs across the country. It is a nationally representative national death cause monitoring network data, which ensures the reliability, accuracy and continuity of the death cause data. Additionally, the data 
spans from 2010 to 2010, it covered the whole country and provided important data for studying the temporal and spatial changes of IHD and the differences between urban and rural areas and gender. Another important strength is that the main risk factor monitoring data comes from the 2013 China Chronic Disease and Risk Factor Monitoring. This monitoring covers 302 monitored counties and districts in 31 provinces (autonomous regions and municipalities). The survey results are both nationally representative and provincial-level representatives. Therefore, from the perspective of data sources, this article uses data from large-scale national surveillance networks, including data on the cause of death, behavioral risk factors, etc., to comprehensively study the relationship between the temporal and spatial changes of IHD and risk factors.

There are limitations in this study. IHD often has many complications, and the chain of disease causes of death is complex and diverse, which makes the judgment of the fundamental cause of death complicated and difficult. At the same time, China has a vast land and abundant resources, the level of economic development is quite different, and a large number of deaths occur at home, which might cause deviations in the judgment of the fundamental cause of death. Therefore, the death data of IHD in this article might be underestimated and misreported. In addition, the quality control of each monitoring point is different, and the quality of the data is different. These potential factors might affect the results of the study to a certain extent.

\section{Conclusion}

Generally, IHD mortality rate increased during 20102015. IHD mortality in the population aged 40 years and over showed significantly differences between gender, urban/rural areas, and regions. The high level of SBP, overweight / obesity (BMI) were risk factors for IHD, while high density lipoprotein (HDL) was a protective factor. Immediate action via primary and secondary preventions to enhance the prevention and management of IHD are required specifically in China. Urbanization rate, medical insurance coverage, and family income were conducive to the reduction in IHD mortality. Measures to further develop economy, improve people's living standards, continue to promote urbanization, increase investment in the medical field, and expand medical insurance coverage were recommended to tackle this crucial health inequality.

\footnotetext{
Abbreviations

IHD: Ischemic Heart Disease; RR: Risk Ratio; Cl: Confidence Interval; BMI: Body Mass Index; SBP: Systolic Blood Pressure; HDL: High Density Lipoprotein; CVD: Cardiovascular Diseases; CHD: Coronary Heart Disease; DALY: Disability Adjusted Life Year; ICD-10: International Classification of Disease 10; DSP: Disease Surveillance Point; GBD: Global Burden Disease; CDRFS: Chronic Disease Risk Factor Surveillance; FBG: Fasting Blood Glucose; MRRs: Median
}

Rate Ratios; PCV: Percentage Change Variance; MOR: Median Odds Ratio; VPC: Variance Partition Coefficient; ICC: Intra-Class Correlation

\section{Acknowledgements}

We would like to sincerely thank Maigeng Zhou, director of the Chronic Disease Center, Jinlei Qi, Yunning Liu, Peng Yin of the Mortality Monitoring Department of the Chronic Disease Center for their participation in data collection and methodological guidance.

\section{Authors' contributions}

BW and PL participated in the design, data collection, data analysis and drafting of the first manuscript. FH and YS participated in the data analysis and manuscript modification. XW and LW participated in the design of the study and review of the draft manuscript. All authors read and approved the final manuscript.

\section{Funding}

This research related work were supported by a Classification of Project:Research on the prevention and control of major chronic noncommunicable diseases (Subject No. 2016YFC1302603). The funding agency had no role in the study design, data collection, data analysis, manuscript writing, or publication

\section{Availability of data and materials}

The datasets used and/or analysed during the current study are available from the corresponding author on reasonable request.

\section{Ethics approval and consent to participate}

This study gained IRB approval, the granting institution was National Center for Chronic and Noncommunicable Disease Control and Prevention, Chinese Center for Disease Control and Prevention. (NCNCD, China CDC).

\section{Consent for publication}

Not applicable.

\section{Competing interests}

The authors declare that they have no competing interests.

\section{Author details}

${ }^{1}$ National Center for Chronic and Noncommunicable Disease Control and Prevention, Chinese Center for Disease Control and Prevention, 27 Nanwei Road, Xicheng District, Beijing 100050, P.R. China. ${ }^{2}$ China and Japan Friendship Hospital, Yinghua East Street 2\#, Chaoyang District, Beijing, P.R. China. Institute of Basic Medical Sciences Chinese Academy of Medical Sciences, School of Basic Medicine Peking Union Medical College, Beijing, China.

Received: 14 July 2020 Accepted: 8 December 2020

Published online: 04 January 2021

\section{References}

1. Ferguson JF, Phillips CM, McMonagle J, et al. NOS3 gene polymorphisms are associated with risk markers of cardiovascular disease, and interact with omega-3 polyunsaturated fatty acids. Atherosclerosis. 2010;211(2):539-44.

2. World Health Organization. Cardiovascular Diseases. Available online: http:// www.who.int/cardiovasculardiseases/en/. (Accessed on 1 March 2018).

3. Shengshou $H$, Runlin $G$, Lisheng $L$, et al. Chinese cardiovascular disease report 2018. Chinese Circ J. 2019;34(3):209-20.

4. Xiaoyun W, Qing Z, Yanrong Z. New developments of multilevel models. J Math Med. 2003;16(2):152-4

5. Gou L, Juying Z, Min W. Application of multilevel statistical models in toxicological research. Chinese J Health Stat. 2002;19:371-2.

6. Hongyan R, Xia W, Cao W. GonghuanY. Spatiotemporal variations in cardiovascular disease mortality in China from 1991 to 2009. BMC Cardiovasc Disord. 2019;19:159

7. Ganshen Z, Chuanhua Y, Maigeng Z, Lu W, et al. Burden of Ischaemic heart disease and attributable risk factors in China from 1990 to 2015: findings from the global burden of disease 2015 study. BMC Cardiovasc Disord. 2018;18:18. 
8. Yang $\mathrm{G}, \mathrm{Hu}$ J, Rao KQ, Ma J, et al. Mortality registration and surveillance in China: history, current situation and challenges. Popul Health Metrics. 2005;3:3.

9. Zhou M, Jiang $Y$, Huang Z, Wu F. Adjustment and representativeness evaluation of national disease surveillance points system. Ji Bing Jian Ce. 2010;25:239-44.

10. Maigeng Z, Linhong W, Shiwei L, Yichong L, Lijun W, et al. Cause-specific mortality for 240 causes in China during 1990-2013: a systematic subnational analysis for the global burden of disease study 2013. Lancet. 2016;387(10015):251-72.

11. Yang GH, Zheng XW, Wang LS, et al. Select of DSP points in second stage and their presentation. Chinese J Epidemiol. 1992;13(4):197-201.

12. Liu YN, Astell-Burt T, Liu JM, Yin P, Feng XQ, et al. Spatiotemporal variations in lung cancer mortality in China between 2006 and 2012: a multilevel analysis. Int J Environ Res Public Health. 2016;13:1252

13. Li $Y$, Wang $L$, Jiang $Y$, Zhang $M$, Wang $L$. Risk factors for noncommunicable chronic diseases in women in China: surveillance efforts. Bull World Health Organ. 2013;91(9):650-60.

14. Leyland AH, Goldstein H. Multilevel Modelling of health statistics. Chichester: Wiley; 2001.

15. Merlo J, Chaix B, Ohlsson H, Beckman A, et al. A brief conceptual tutorial of multilevel analysis in social epidemiology: using measures of clustering in multilevel logistic regression to investigate contextual phenomena. J Epidemiol Community Health. 2006;60:290-7.

16. Browne WJ, Subramanian SV, Jones K, Goldstein H. Variance partitioning in multilevel logistic models that exhibit overdispersion. J R Stat Soc. 2005;1: 599-613.

17. Merlo J, Yang M, Chaix B, et al. A brief conceptual tutorial on multilevel analysis in social epidemiology: investigating contextual phenomena in different groups of people. J Epidemiol Community Health. 2005;59:729-36.

18. Rasbash J, Steele F, Browne W, Goldstein H, et al. A user's guide to MLwiN version 2.33. London: Multilevels Models Project, Institute of Education, University of London; 2004. p. 134-6.

19. Cui Y, Zhu Q, Lou C, Gao E, Cheng Y, Zabin LS, et al. Gender differences in cigarette smoking and alcohol drinking among adolescents and young adults in Hanoi, Shanghai, and Taipei. J Int Med Res. 2018;46(12):5257-68.

20. Jibin T, Xiumin Z, Weihua W, Peng Y, Xiaomin G, Maigeng Z. Smoking, blood pressure, and cardiovascular disease mortality in a large cohort of Chinese men with 15 years follow-up. Int J Environ Res Public Health. 2018; 15:1026.

21. Li W, Gu H, Teo KK, Bo J, Wang Y. Et al; PURE China investigators. Hypertension prevalence, awareness, treatment, and control in 115 rural and urban communities involving 47000 people from China. J Hypertens. 2016;34(1):39-46

22. Zhang $X$, Khan AA, Haq EU, Rahim A, et al. Increasing mortality from ischemic heart disease in China from 2004 to 2010: disproportionate rise in rural areas and elderly subjects. 438 million person-years follow-up. Eur Heart J. 2017;3:47-52.

23. Nowbar AN, Howard MGJP, Francis DP, et al. Mortality from ischemic heart disease. Circ Cardiovasc Qual Outcomes. 2019;12:e005375.

24. Laekland DT, Roece EJ, et al. Factors influencing the decline in stroke mortality: a statement from the American Heart Association/American Stroke Association. Stroke. 2014;45(1):315-53.

25. Lv J, Yu C, Guo Y, Bian Z, Yang L. Et al; China Kadoorie biobank collaborative group. Adherence to healthy lifestyle and cardiovascular diseases in the Chinese population. J Am Coll Cardiol. 2017;69(9):1116-25.

26. Rajeev G, Salim Y. Challenges in management and prevention of ischemic heart disease in low socioeconomic status people in LLMICs. Gupta Yusuf BMC Med. 2019;17:209.

27. Fontecha J, Calvo MV, et al. Milk and dairy product consumption and cardiovascular diseases: an overview of systematic reviews and metaanalyses. AdvNutr. 2019;10:S164-89.

28. Miller V, Mente A, Dehghan M. Fruit, vegetable, and legume intake, and cardiovascular disease and deaths in 18 countries (PURE): a prospective cohort study. Lancet. 2017;390:2037-49.

29. Weiwei C, Hui S, Liyuan M. Current situation and progress of prevention and treatment of cardiovascular and cerebrovascular diseases in China. Prev Treat Cardio-Cerebral-Vasc Dis. 2016;16(2):79-83.

30. Shin J, Ham ID, Shin S, Choi SK, Paik H-Y, Joung H. Effects of lifestyle-related factors on ischemic heart disease according to body mass index and fasting blood glucose levels in Korean adults. PLoS One. 2019;14(5):e0216534.
31. Kwon $\mathrm{CH}$, Kang J, Cho A, et al. Optimal target blood pressure and risk of cardiovascular disease in low-risk younger hypertensive atients. Am J Hypertens. 2019;32(9):833-41.

32. Moon JH, Koo BK, Moon MK. Optimal high-density lipoprotein cholesterol cutoff for predicting cardiovascular disease: comparison of the Korean and US National Health and nutrition examination surveys. J Clin Lipidol. 2015;9:334-42.

33. Møller SV, Hannerz H, Hansen ÅM, et al. Multi-wave cohort study of sedentary work and risk of ischemic heart disease. Scand J Work Environ Health. 2016:42(1):43-51.

34. Biswas A, Oh Pl, Faulkner GE, Bajaj RR, et al. Sedentary time and its association with risk for disease incidence, mortality and hospitalization in adults: a systematic review and meta-analysis. Ann Intern Med. 2015. 162(2):123-32.

35. Zhixiong $Z$, Zenong $Y$, Binsheng $Y$, et al. Relationship between sedentary and less active behavior and cardiovascular risk factors in working population. Natl Sports Sci Conference. 2015:738-9.

\section{Publisher's Note}

Springer Nature remains neutral with regard to jurisdictional claims in published maps and institutional affiliations.

Ready to submit your research? Choose BMC and benefit from:

- fast, convenient online submission

- thorough peer review by experienced researchers in your field

- rapid publication on acceptance

- support for research data, including large and complex data types

- gold Open Access which fosters wider collaboration and increased citations

- maximum visibility for your research: over $100 \mathrm{M}$ website views per year

At $\mathrm{BMC}$, research is always in progress.

Learn more biomedcentral.com/submissions 